\title{
Analysis of Symbolic Meanings of Folk Paper-cut from the Perspective of Semiotics
}

\author{
Shen Tao \\ Hezhou University, Hezhou City, Guangxi Province of China \\ E-mail: bclb7372@sina.com
}

\begin{abstract}
Chinese folk paper-cut is an artistic sign of materialization. This art is the sign associated with the creator's certain expressive meanings. The artistic creation for the folk paper-cut is the process from a complete signifier (the form of a sign) to the higher-level signified (the concept or object that's represented) of complex meanings. This means that paper-cutting art possesses the symbolic system attributes of artistic expression media. The application of symbolic sign language in paper-cut not only highlights the cultural nature of folk art, but also builds a rich semiotic system for the folk paper-cut.
\end{abstract}

Keywords-Folk paper-cut; Signified; Signifier; A semiotic system

\section{A SEMIOTIC SYSTEM OF FOLK PAPER-CUT}

Signs refer to the carrier form perceived by mankind and representing meanings, as well as an intermediary or a tool that connects material and consciousness. As a culture product, a sign does not denote any real thing but acts as an implied meaning [1]. Ferdinand de Saussure said, "We call signs the combination of concepts and sound-images, use the signified and the signifier to replace concepts and sound-images respectively. Saussure holds that any linguistic sign consists of both the signifier and the signified, "the former" meaning images, "the latter" concepts, so a sign is a mixture of the signifier and the signified. Roland Barthes concluded: "Like modes of linguistic signs, semiotic signs are also mixtures of the signified and the signifier."[2] If there is no natural connection between the signifier and the signifier of signs, their connection is to conventionally follow "the arbitrary principle of signs," we can not individually explain the notion of each sign, and a semiotic system composed of conventional principles must be established. Only in this way can we explain all signs from this system.

Paper cutting, originating in ancient religious worship, possesses a strong symbolic meanings and subjective colors. It has very stable theme expression, such as the paper-cut content having always been the performance of real life; the theme choice is also consistent, such as the constant pursuit of auspicious themes. This seems to indicate that there is some unified and clear "creative norms", which shows that a complete set of concept about the sign signifying system lies in paper-cut. This concept subtly and conventionally influences paper-cutting creation of folk artisans. These stable and convergent characteristics of paper-cut must have foreshadowed the existence of an accurate sign system for paper-cutting art. With regards to the semiotic system, Saussure made an example, "when we replace "word" with "term", a system concept is established," [3]. When due to some kind of internal or external relationship, a variety of signs combine as one, that is, a semiotic system.

\section{THE SIGNIFYING SYSTEM OF FOLK PAPER-CUT SIGNS}

The sign concept of paper-cut creation should be composed of collective and individual consciousness. As the inheritance of the collective wise consciousness of ancient people in previous generations, the collective consciousness makes people apply the objective images connected with life into the paper-cut creation and gradually turns these images into a substitute for expressing the specific sign concepts of paper-cut For instance, the lotus means harmony and happiness; the pomegranate and grape many children bringing much happiness; the crane longevity; the goldfish wealth. These plants and animals do not represent their actual prototypes, but have become substitutes for people's consciousness and conception. This kind of contract substitutes for social collective consciousness is a semiotic system that can not be arbitrarily changed by individuals. For example, paper-cut decoration for rural bridal chambers the peony and moon indicate perfect conjugal bliss; the magpie and plum great happiness; the kylin and auspicious clouds children delivered by kylin. Women who create paper-cut first have these good wishes of collective consciousness like signified ideas of perfect conjugal bliss, great happiness and children delivered by kylin, and then conceive their many signifiers, that is, when the collective consciousness of the peony and moon, the magpie and plum, as well as the kylin and auspicious clouds exists first, all the patterns used for decorating bridal chamber can be cut out. However, in the practical creation, the inherited collective consciousness signs do not end up as the artistic images exhibited by the paper-cut works. On the one hand, signs of collective consciousness lack individualized connotation. On the other hand, those individuals who construct the form of paper-cut art are all the conceptual carriers with current awareness. Thus the creativity performance of individual consciousness begins to play a role.

Therefore, in the creative process of paper-cutting, the signifier's meanings of individual consciousness is far more important than the collective consciousness. Every paper-cut artist has his own aesthetic taste and emotional sustenance, because individuals are faced with different realistic problems. 
Although regional styles of paper-cut influenced by individual consciousness are different, all directly originate from much the same traditional folk subjects, and obey the traditional contract that the individual can not casually transform easily. Hence, the folk paper-cut does not simply imitate natural images, but emphasizes to express the realistic feelings of individuals. For example, with a piece of red paper folded into several layers, the women scissor the paper some times, and then unfold it, the paper taking shape with obvious individual style characteristics. The process of cutting is of strong expressiveness, which surpasses the specific images of papercutting subjects. The women do not follow samples to create paper-cut, but show paper-cut creation on the basis of their own direct senses of life with obvious originality. This is why under the same subject, with different regions and creators paper-cut can be of various expressions. Therefore, when an individual begins to create his expression needs, he not only integrates the signified meanings of signs through subjective consciousness, but also makes an active selection of signifiers shapes of signs.

\section{SEMIOTIC MEANINGS OF FOLK PAPER-CUT}

During artistic creation, people are used to pursuing certain manifestations of "meanings"; they also like to explain those conveyed meanings in artistic exchanges. When the interpretation of signs achieves exchanges and responses in constant collision, the meaning of signs emerges from the interaction appropriate for artistic dissemination. As soon as the folk paper-cut signs are integrated into a system in the corresponding history and practice, the artistic value of the signs will be realized through exchanges and interactions. If any inconsistency appearing between disseminators and receivers causes no interaction, the signs will lose their meanings. In each round of dissemination, people inject their own revised or rich interpretations into signs, which leads to new developed "meanings". The earlier obscure meanings of paper-cutting signs become increasingly clear by means of continuous elucidation and enrichment, thereby making the signified combination of paper-cut meanings become more and more verifiable in creation.

When collective consciousness and individual consciousness act on the paper-cut creation, the signified and signifier will form a semiotic system of paper-cut art, which is Susanne Langer's "the signs in art" and "the art signs" [4]. Susanne Langer employed the meaning system named "the signs in art" which belong to the signs of universal meanings to serve as the media of expression or carriers of meanings. This system is defined by the transcendental concept. "The art signs" are a creative system which is rebuilt with "the signs in art" as the carrier, belonging to the individualized and expressive symbols of emotions and views. Of course, the semiotic system of paper-cut is not a simple correspondence between the signifier and the signified but an integrated papercutting system consisting of various representing components and multi-level represented components. In terms of the image of the magpie, two magpies mean good things coming in pairs; the magpie and badger mean boundless joy; the magpie and leopard represent good news; the magpie and lotus mean admission to great schools. Corresponding represented components' multi-level and complex meanings depend on the combination of paper cutting subjects, and the more complex the combination is, the more levels the extended meaning of the represented component will have. Using the concept of semiotics to analyze the folk paper-cut's signs will help us understand the general characteristics and the shaping rules of folk paper-cut.

\section{SYMBOLIC MEANINGS OF THE FOLK PAPER-CUT SEMIOTIC SYSTEM}

Pierce, from the perspective of the meaning relationship between signs and objects, classifies signs into: "an icon, an imitative or similar relationship between the signifier and the signified, an index, an internal causal relationship between the signifier and the signified that need to be understood by the association in mind, and a symbol, an arbitrary non-necessary internal relationship between the signified and the signifier as well as requiring explanations to reveal the sign meaning."[5]. In fact, the application of the symbolic meanings of the semiotic system in folk paper-cut can make the complex and abstract concepts simple and vivid. This application can also extend the internal implications of paper-cut's forms, thereby creating an artistic conception to arouse people's imagination and enhance the expressiveness and artistic effects of paper-cut. Therefore, the symbolic meanings of the folk paper-cut signs are manifested in at least three aspects.

Firstly, by virtue of analogy or association, folk paper-cut's themes and contents obtain the symbolic meanings of the "meaning". For example, people repeatedly make paper-cut inheriting the same subjects in all ages, because more "meaningful" symbolic meanings often exist behind these traditional paper-cut themes. Therefore, paper-cut originates from a traditional form of the worship of nature and religions from mankind, but then become a kind of symbolic meaning with many beautiful aspirations through evolutions. Hence, people tirelessly extend the conventional "meaning" to a newer and deeper symbolic meaning, making it more cultural and social, and gradually become a fixed semiotic system at last. For instance, a large amount of paper-cuts with the themes of prosperity brought by the dragon and the phoenix, and children delivered by kylin across the China is to convey blessings of the prosperity of people and a good luck instead of bad one.

The second is the symbolic meaning conveyed by patterns of folk paper-cut. The patterns of folk paper-cut generally refer to the appearances and structures of the objects represented by "shapes". Traditional folk paper-cut patterns are rooted in the source of traditional Chinese art which is influenced by Chinese characters and regions. The main focus is on the decorative performance of the patterns, the alternation and space connection relationship between real and imaginary shapes. In addition, during the construction, follow the pursuit of the complete, symmetrical, and homogeneous forms. For example, the patterns of five blessings around a longevity, four joyful dolls, double happiness brought by a dragon and a phoenix, and a bumper grain harvest are in this form. Moreover, some fixed structure models loved by people, such as the lotus, the boy, the horse and the monkey in the patterns of the "son born in lotus" and "monkey on horse", are no longer simply imitating certain images in the nature. They are formalized by 
the combination of traditional beliefs and utilitarian aspirations, and have distinctive beliefs and symbolic meanings.

Furthermore, the color concept of traditional paper-cut is deeply influenced by Chinese ethical and moral values and national aesthetic consciousness. Generally, there are two types of paper-cut colors, namely the single color paper-cut and the multiple color paper-cut. For the single color paper-cut, red is the most common. Different colors of paper-cut express different concepts, such as the red paper-cut is festive, auspicious and blessed; the green represents longevity; the yellow and black expels disasters and evil spirits; the indigo and white paper-cuts is often used in funeral activities, expressing grief and sadness. This color concept influences the folk artists imperceptibly and is inherited through successive generations, constituting an important color language of the folk paper-cut. The multiple color paper-cut, also known as the chromatic color paper-cut, is collaged by a number of cut pieces of colored paper, or dyed from the cut white paper, or cut into a template on a piece of white paper and then dyed with a variety of colors. Generally, application of the colors of the multiple color paper-cut emphasizes the proportion of colors, purchasing coordination in the color contrasts, and highlighting subjectivity and the symbolic meanings of the colors. Once a color conflict can be felt, put the colors onto a black paper, you will get a bright and coordinate feeling. If you line colors with golden strands, equally satisfactory results will come into being. For example, folk paper-cut artists in Fujian and Guangdong regions use tin or copper to carve patterns lined by the colored paper to create a splendid and magnificent experience. In addition, the folk paper-cut artists focus on contrasts between the warm and cold colors when they design colors, and in this way they will use color match to create a warm and peaceful visual aesthetic effect.

\section{CONCLUSION}

Folk paper-cut is an important part of folk visual arts, a product of human's material and spiritual life, and the most direct expressive form of the purest artistic thoughts and language of people's labor life. The reason why paper-cut of various themes prevailing in various places of China is that paper-cut has the meaning of exorcising evil spirits and things, protecting lives and children, and promoting longevity. Some utility aim can be achieved by means of intuitive and concrete forms or behaviors. Because the things that folk paper-cuts represents are signs with specific symbolic meanings, the image and structure of paper-cut does not indicate the meaning of the real thing, just as the women who create paper-cut repeatedly say, "flowers have no colors and birds no names, so just take the meaning." It shows that the fundamental purpose of folk paper-cut is to "take the meaning," and attention is paid to the subjective symbolic meaning signs formed by the papercut images. Hence, in paper-cut, we find the pursuit of the Chinese folk art, that is, Chinese people does not care about mechanical imitation "colors or names" of natural concrete objects, and we also understand the origin and significance of the interpretability of the folk paper-cut's semiotic system. Since the inheritance and the development of folk paper-cut has a highly universal, open and long-lasting foundation, folk paper-cut is bound to become a kind of authentic public art, and it inevitably becomes the effective way for the majority of people to express their art about real world.

\section{REFERENCES}

[1] (Switzerland) written by Saussure, translated by Gao Mingkai. "General Linguistics Course". [M] Beijing: the Commercial Press, 1980-11: 102

[2] (France) written by Roland Barthes, translated by Dong Wenxue and Wang Kui. "Semiotic Aesthetics". [M] Liaoning: Liaoning People's Publishing House, 1987-9: 36

[3] Xu Peng. "Preliminary Study of Peirce's General Semiotics" [J]. Journal of Yunnan University (Social Science Edition), 2007-01: 23-29 + $66+$ 94.

[4] (United States) written by Susan Langer, translated by Teng Shouyao and Zhu Jiang. "Art Issues". [M] Beijing: China Social Sciences Publishing House, 1983-3: 133-134

[5] Wang Zhiliang. "Semiotics and Visual Art of Pierce" [J]. Journal of Nanjing University of the Arts (Arts \& Design), 2011, 03: 108-114. 